\title{
Physical and Psychological Vulnerability of Adolescents during Pregnancy Period as Well as Post Traumatic Stress and Depression after Child Birth
}

\author{
Masuma Akter \\ Fareast International University, Dhaka, Bangladesh \\ Email: soniabd1@hotmail.com
}

How to cite this paper: Akter, M. (2019) Physical and Psychological Vulnerability of Adolescents during Pregnancy Period as Well as Post Traumatic Stress and Depression after Child Birth. Open Journal of Social Sciences, 7, 170-177.

https://doi.org/10.4236/jss.2019.71015

Received: November 30, 2018

Accepted: January 15, 2019

Published: January 18, 2019

Copyright $\odot 2019$ by author(s) and Scientific Research Publishing Inc. This work is licensed under the Creative Commons Attribution International License (CC BY 4.0).

http://creativecommons.org/licenses/by/4.0/

(c) (i) Open Access

\begin{abstract}
This study attempts to examine the physical and psychological vulnerability of adolescents during pregnancy period as well as post traumatic stress and depression after child birth. Pregnancy and childbirth among adolescents has become a very common issue nowadays which has been developing physical as well as psychological vulnerability among the teenagers. Most of the time, young mother has to suffer from severe health problems and complications. There is high health risk for the new born baby and they are more likely to suffer from several types of infections and diseases. Most often, teenage mothers do not get support and proper care from their parents which are more likely to increase the prenatal health risks for young mothers. Moreover, prenatal care has significant benefits for both baby and mother in order to monitor mother's health condition, baby's growth and quick response towards any complications that can be arisen at any time. High blood pressure, hyper tension, preeclampsia etc. are very common physical problems of the teenage mothers. These health risks badly affect the teen mothers and they need to take medication which may disrupt baby's growth. Moreover, it has tremendous long term psychological effect on both mother and children. Young mothers go through several mental changes and face so many new challenges, like sleepless night, depression, shortage of time to take proper care of own self and so on. This study attempts to find out the major physical and psychological consequences of adolescents during pregnancy period as well as after child birth.
\end{abstract}

\section{Keywords}

Adolescents, Physical Vulnerability, Psychological Vulnerability, Mental Stress 
and Depression

\section{Introduction}

Adolescence indicates the period of growing up. It is normally an important period of girl's physical and mental changes. During the adolescence stage, biological and psychological developments take place. Puberty, the important landmark of sexuality, is developed during this period. Adolescence is a phase of transition during which major developments of sexuality take place. The hypothalamo-pituitary-gonadal axis function is highly essential for the sexual development during puberty [1].

Adolescence can be broadly divided into three stages: early (10 - 13 years), middle (14 - 16 years), and late (17 - 19 years). Physical changes start in early adolescence, where they are very concerned about their body image. During adolescence, cognitive development takes place; adolescents develop abstract thinking and reasoning. Emotionally, they develop a sense of identity during late adolescence; social involvement, peer interaction, as well as sexual interest, develop in this phase. Different behavioral experimentation is seen in early adolescence, and risk takes in middle adolescence, and later adolescents learn to assess their own risk taking [2]. Teenage pregnancy and adolescent fatherhood results from early exposure to sexual relationship. In some families, sexuality is discussed little, so there is little scope to explore sexuality, and it still remains as a myth or enigma for the adolescent, which affects their perception of sexuality [1]. During adolescence, an individual's need for intimacy and love making with opposite gender increases. Adolescents explore about different appropriate ways to express the love and intimacy. The development of an adolescent occurs not in isolation, rather in the background of the family, society in a defined culture, which significantly influences the adolescent sexuality [3]. Statistics indicates that pregnant teenagers are more likely to terminate the pregnancy than proceeding with the birth. However, while childbirth is the outcome, long term negative implications of teenage pregnancy are considerable. Teenage births carry a higher risk of complicated pregnancies, low birth-weight, premature births and the need for neonatal intensive care. Negative consequences come at considerable cost to mother and child, their families and the wider community. Teenage pregnancy poses a risk in which the victims have little or no control over critical social, health, and economic outcomes and as a result, they are socially isolated without sufficient knowledge and skills to effectively navigate life's challenges. It can also be seen as an early warning sign of inequality in a society [4].

\section{Methodology}

In this study, I propose to use qualitative method to gather data. Participant observation, case study and interview method have been used to collect required 
data.

\subsection{Literature Review}

For millions of young people around the world, the onset of adolescence brings not only changes to their bodies but also new vulnerabilities to human rights abuses, particularly in the arenas of sexuality, marriage and childbearing. Millions of girls are coerced into unwanted sex or marriage, putting them at risk of unwanted pregnancies, unsafe abortions, sexually transmitted infections (STIs) including HIV, and dangerous childbirth. Adolescent boys are at risk, as well. Young people-both boys and girls-are disproportionately affected by HIV [5]. Girls who are vulnerable are more likely to become pregnant. In every region of the world-including high-income countries-girls who are poor, poorly educated or living in rural areas are at greater risk of becoming pregnant than those who are wealthier, well-educated or urban. This is true on a global level, as well: 95 per cent of the world's births to adolescents (girls aged 15 - 19) take place in low- and middle-income countries. Every year, some 3 million girls in this age bracket resort to unsafe abortions, risking their lives and health. Girls, who lack choices and opportunities in life, or who have limited or no access to sexual and reproductive health care, are more likely to become pregnant. Girls forced into child marriage - a violation of their human rights-are also more likely to become pregnant. In developing countries, nine out of 10 births to adolescent girls occur within a marriage or a union [6]. In most places in the world, girls' first sexual activity occurs during late adolescence, between the ages of 15 and 19. Human papillomavirus (HPV) affects relatively young women, nearly 90 percent of them in developing countries, and is the largest single cause of years of life lost to cancer in the developing world. Childbearing adolescents are susceptible to a myriad of health-related problems. For these youth, health-related problems include both physiological and psychological disease states that negatively impact both of their own and children's lives. Suicide attempts, parenting problems (including child abuse), domestic violence, poor birth outcomes, and sexually-transmitted diseases (including HIV) are just some of the problems encountered [7]. Gestational hypertension, or high blood pressure caused by pregnancy, can cause premature delivery or low birth weight of the baby. When high blood pressure develops into a life-threatening condition called preeclampsia, both mother and unborn baby are at risk. This sudden increase in blood pressure after the 20th week of pregnancy is not preventable and requires very close monitoring by an obstetrician [8]. Tens of thousands of adolescents die annually of causes related to pregnancy and childbirth. In fact, these are the leading cause of death among adolescent girls, aged $15-19$, globally. Health problems are even more likely if a girl becomes pregnant too soon after reaching puberty. In lowand middle-income countries, the risk of maternal death for girls under age 15 is higher than for women in their twenties. These girls also face health risks such as obstetric fistula, and their babies face greater risks as well [6]. Approximately 14 
percent of pregnant women develop anemia, and the condition occurs in higher rates in pregnant adolescents because of "the insufficient amount of healthy caloric intake needed during pregnancy" as well as "the increased iron requirements associated with the expansion of the red cell mass during adolescence." More pregnant 15- to 19-year-olds develop anemia than pregnant women from 20 to 44 . It's imperative for teenage girls to seek out qualified prenatal care to ensure they are receiving an adequate intake of nutrients and prenatal vitamins to prevent iron and other nutritional deficiencies [8].

Few risks that are greater at the teen pregnancy are:

- low birth weight/premature birth

- anemia (low iron levels)

- high blood pressure/pregnancy induced hypertension, PIH (can lead to preeclampsia)

- a higher rate of infant mortality (death)

- possible greater risk of cephalopelvic disproportion (the baby's head is wider than the pelvic opening) [9].

Maternal stress during pregnancy increases the risk of the child having a range of altered neurodevelopment outcomes. The stress can be of different types, and at least for some outcomes, there seems to be a linear dose response effect. Not all children are affected, and those that are, are affected in different ways. The gestational age of vulnerability probably differs for different outcomes [10]. Among adolescent mothers, rates of depression are estimated to be between $16 \%$ and $44 \%$. In contrast, the lifetime prevalence of major depression among non-pregnant adolescents and adult women is between 5\% and 20\% [11]. Depression symptoms among young mothers are also more likely to persist well after the birth of their child [12]. Although there are few prospective, longitudinal studies on the long-term mental health outcomes of adolescent mothers, 1 study of African American adult women who became mothers during adolescence found a twofold increase in depression 20 years after the birth of their first child [13]. While postpartum depression can affect up to 20 percent of mothers, the prevalence of postpartum depression (or related postpartum mood disorders, i.e. postpartum blues or and postpartum psychosis.) for teen mothers is nearly double, "a fact that most people don't even know", says Gloria Malone, a writer, activist, and consultant, who was 15 when she had her daughter. "I was happy but I was tired, overwhelmed, and felt like I had no one to talk to because everyone seemed too busy putting me down... she wrote in a blog post". I needed someone to talk to and the extreme isolation. I felt due to society and people pushing me away and down kept me from finding someone to speak to [14].

\subsection{Research Objective}

1) To find out the major reasons why adolescents are more venerable during pregnancy period.

2) To find out the significant health risks and other physical complications 
during the whole pregnancy period.

3) To get the information about the after effect of adolescence pregnancy including physical as well as mental hazards.

4) To find out the probable psychological stress and trauma during teenage pregnancy period.

5) To find out how does prenatal stress interact with genetic vulnerabilities?

6) To find out how are the effects of prenatal stress moderated by the nature of the postnatal care?

7) To find out the effective solutions of the prevailing situation.

\section{Result of the Study}

Adolescents face a great challenge in their early stage. Major biological, as well as psychological changes, occur during this period. A huge number of teenagers become pregnant each year, and $85 \%$ of these pregnancies are unplanned. Pregnancy mainly occurs at the first time sexual intercourse without the use of contraception. Generally the teenagers do not have much knowledge about contraceptive methods and they are afraid of using contraceptives because of some common fear like weight gaining, future problems with pregnancy etc. Sexually transmitted diseases (STDs) can be transmitted during first intercourse. Sexual exposure during adolescence is a matter of serious concern due to the risk of transmission of sexually transmitted infections including HIV infection/AIDS, teenage pregnancy, and adolescent fatherhood. Early sexual exposure and pregnancy also has adverse consequences on the reproductive health. It also increases the risk of sexually transmitted diseases. The life of adolescents changes rapidly due to their pregnancy. Complications from pregnancy and childbirth are the leading cause of death among adolescent girls. Teen's pregnancy also associated with malnutrition and poor health condition. Most of the time the teen pregnant mothers do not get sufficient calories, protein, carbohydrates, vitamins or minerals during the whole pregnancy period and thus suffer from malnutrition along with some other health sickness like dizziness, poor energy level, hypertension, low blood pressure and so on. Childbearing adolescents have to suffer from several health-related problems. Adolescent Pregnancy may lead devastating health consequences for girls. Most of the time adolescents are not physically ready for pregnancy or childbirth thus they are more vulnerable to complications. Health-related problems include both physiological and psychological disease which has negative impacts both of their own and their children's lives. Most of them are nutritionally depleted. Sexually-transmitted diseases, Suicide attempts poor birth outcomes, parenting problems are the major problems among the childbearing adolescents. The chances of premature birth are high in teenage pregnancies. Premature birth may accompany several short-term as well as long-term medical complications, such as respiratory concerns, digestive problems, vision issues and cognitive disorders. High blood pressure is a very common problem among teenage adolescents. High blood pressure during tee- 
nage pregnancy is also linked to organ damage and other health problem such as preeclampsia, which disrupts foetal growth. Low-birth-weight Baby-Preemies, babies born before 40 weeks of pregnancy term, are more likely to weigh less (1500 - 2500 grams). The reason for low birth-weight of a baby is the insufficient time in the womb for development. The major risks are:

- The chances of pre-term birth are high in teenage pregnancies.

- Pregnant teens are at a higher risk of high blood pressure condition.

- Lack of prenatal care is another danger of teenage pregnancy.

- Postpartum depression rate is higher in teenage mothers.

Most of the time teens don't get prenatal care. Pregnant teens and their babies are at higher risk of health problems than older pregnant women. They are vulnerable to anemia, or low blood iron, and pregnancy-related high blood pressure. Severe tiredness and other complications may occur due to anemia. Preeclampsia caused by high blood pressure is another life-threatening condition for both mother and unborn baby. Tendency of drinking, smoking, taking drugs in adolescent's pregnancy period is very common which may increase the risk of having a low-birth-weight baby. Complications in pregnancy, premature birth and stillbirth may occur due to severe smoking. In most of the cases premature baby may suffer from the following health problem:

- Cerebral palsy

- Breathing problems and asthma

- Feeding difficulties

- Serious intestinal problems

- Developmental delay

- Mental retardation

- Bleeding in the brain

- Vision problems or blindness

- Hearing problems

- Sudden infant death syndrome (SIDS)

Teen mothers are more likely to face several social and psychosocial stresses and become the victim of mental health problems. Sometimes the responsibilities and demands of parenting seem burden to them and they fail to cope up with the new situation. Such type of stress may create lots of mental or psychological problems among the teen mothers. These sorts of psychological problems hamper their normal parenting behavior. Additionally, the adolescent mothers experience higher rates of depression than adult mothers. The life of adolescents changes rapidly due to their pregnancy. Her education and job prospect may diminish due to their early pregnancy. Adolescent pregnancy has negative effects on girl's education and income-earning potential. Girls who become pregnant at the early stage are pressured or forced to drop out of school. It becomes very hard for teenage mother to continue their further studies. They are less likely to have a job or economically independent. Moreover they are more likely to live in poverty and are at risk of domestic violence. Children of teens are more likely to 
drop out of school, exposure to domestic abuse and violence. Postpartum Depression-Postpartum depression rate is higher in teenage mothers in comparison with other mothers. It may interfere with newborn baby care and his development. Adolescent mothers are more likely to the victim of physical and sexual abuse. Moreover, they have poor access to quality health care resources. Untreated depression may increase the risk of suicidal tendency and attempts. Postpartum stress and disorder arise because of negative attitudes from the society. Most often the teens even do not get proper care as well as support from their family members. Moreover majority of them suffer from financial crisis. Responsibility of parenting and early child bearing may often increase their depression.

\section{Conclusions}

Teenage pregnancy has become an alarming situation now and it has devastating effects on the teen's life. It has direct impact on both young mothers and their newborn babies. Most often, the teen mother does not go for regular prenatal checkups. Thus, the risk of health complication increases at an alarming rate. Moreover, lack of proper prenatal care often induces several medical complications. Moreover, education of the teen mother remains on hold due to their pregnancy and they become unable to continue their further studies due to their responsibilities towards their new born babies. Moreover, the social life of the teen mother gets ruined due to her early and unexpected pregnancy, and she has to spend her life in mental trauma. Additionally, they also suffer from emotional trauma due to lack of social support from family. Having a child at a young age hampers to fulfill their dreams. The young parents most often suffer from severe depression due to facing the negative feedback about the pregnancy from the society. In such situation, suicide attempts and attempting to self-abort the baby are very common scenarios. Some effective measures and steps are required to avoid early pregnancy among adolescence. We need to ensure adolescents' access to sexual and reproductive health information to make them aware of the regarding issue. We need to prevent child, early and forced marriage, sexual violence, and coercion. Some other steps that can be taken are given as follows:

- Easy access to accurate sexual and reproductive health information;

- Availability of safe and affordable contraceptive methods;

- Counseling and social awareness that can be effective;

- Ensure proper care for all pregnant women and girls;

- The prevention and management of sexually transmitted infections;

- To ensure girls right and women empowerment.

Prenatal care and healthy diet including folic acid, calcium, iron, protein and other essential nutrients for both mother and baby is highly required.

\section{Conflicts of Interest}

The author declares no conflicts of interest regarding the publication of this paper. 


\section{References}

[1] Sujitr, K.K., Ananya, C. and Abhishek, S.P. (2015) Understanding Normal Development of Adolescent Sexuality: A Bumpy Ride. Journal of Human Reproductive Sciences, 8, 70-74. https://www.ncbi.nlm.nih.gov/pmc/articles/PMC4477452

[2] Resource Centre for Adolescent Pregnancy Prevention. (2003) An Overview of Adolescent Development Education Training and Research Associate. http://www.etro.rg/recapp/theories/adolescent development/overview.htm

[3] Ott, M.A. (2010) Examining the Development and Sexual Behavior of Adolescent Males. Journal of Adolescent Health, 46, S3-S11. https://doi.org/10.1016/j.jadohealth.2010.01.017

[4] Vulnerability of Teenage Girls to Pregnancy in Ibarapa Central Local Government Area, Oyo State, Nigeria. http://vc.bridgew.edu/cgi/viewcontent.cgi? article $=1889 \&$ context $=$ jiws

[5] Adolescent Sexual and Reproductive Health. UNFPA, United Nations. https://www.unfpa.org/resources/adolescent-sexual-and-reproductive-health

[6] Adolescent Pregnancy. UNFPA, United Nations Population Fund. https://www.unfpa.org/adolescent-pregnancy

[7] Lesser, J. and Escoto-Lloyd, S. (1999) Health-Related Problems in a Vulnerable Population: Pregnant Teens and Adolescent Mothers. Nursing Clinics of North America, 34, 289-299. https://www.ncbi.nlm.nih.gov/pubmed/10318724

[8] Risks of Teenage Pregnancy. Revere Health, Live Better. https://reverehealth.com/live-better/risks-teen-pregnancy

[9] Teen Pregnancy Issues and Challenges. American Pregnancy Association. http://americanpregnancy.org/unplanned-pregnancy/teen-pregnancy-issues-challen ges

[10] Teen Pregnancy Issues and Challenges. American Pregnancy Association. http://americanpregnancy.org/unplanned-pregnancy/teen-pregnancy-issues-challen ges

[11] Kessler, R.C. (2003) Epidemiology of Women and Depression. Journal of Affective Disorders, 74, 5-13. https://doi.org/10.1016/S0165-0327(02)00426-3

[12] Boden, J.M., Fergusson, D.M. and John Horwood, L. (2008) Early Motherhood and Subsequent Life Outcomes. Journal of Child Psychology and Psychiatry, 49, 151-160. https://doi.org/10.1111/j.1469-7610.2007.01830.x

[13] Deal, L.W. and Holt, V.L. (1998) Young Maternal Age and Depressive Symptoms: Results from the 1988 National Maternal and Infant Health Survey. American Journal of Public Health, 88, No. 2. https://doi.org/10.2105/AJPH.88.2.266

[14] Teen Moms Are More Likely to Get Depressed. Tonic. https://tonic.vice.com/en_us/article/mg9pqq/teen-moms-are-more-likely-to-get-de pressed 